\title{
Scheduled-Links Multicast Routing Protocol in MANETs
}

\author{
Hasan Abdulwahid ${ }^{\mathrm{a}}$, Bin Dai ${ }^{\mathrm{a}}$, Benxiong Huang ${ }^{\mathrm{a}}$, and Zijing Chen ${ }^{\mathrm{a}, \mathrm{b}}$ \\ ${ }^{a}$ School of Electronic Information and Communication, Huazhong University of Science and Technology, Wuhan, 430074, China \\ ${ }^{b}$ Centre for Quantum Computation and Intelligent Systems, Faculty of Engineering and Information Technology, University of Technology, Sydney, Ultimo, NSW
} 2007, Australia

\begin{abstract}
Dynamically changing of network topology due to node movement is considered a main challenge in providing reliable and lowcost paths in one to many ends of communication in mobile ad hoc networks. To address this challenge, this paper proposes a scheduled-links multicast routing protocol (SLMRP) based on mobility prediction. SLMRP constructs multiple scheduled paths between multicast sources and receivers. SLMRP scheduled paths are subject to reliability and quality of service requirements in load-balance strategy. Multiple loop-free and node-disjoint paths are constructed for each source-receiver pair during route discovery process. To control the activation and deactivation of these paths, we introduce multicast routing activation timer (MRAT) and path timeout timer (PTT). MRAT and PTT are computed according to the route expiration time for the set of the discovering paths. Performance comparison results demonstrate that the proposed protocol outperforms PUMA, MAODV, and PMRP.
\end{abstract}

Keywords: Scheduled-link, Multicast routing activation timer, Path timeout timer, Wireless networks.

\section{Introduction}

Mobile ad hoc networks (MANETs) are autonomous system$\mathrm{s}$ that comprise a collection of mobile nodes that use wireless transmission for communication. They are self-organized, selfconfigured, and self-controlled infrastructure-less networks [1]. Dynamic changing of network topology due to node movement is considered the most prominent feature of MANETs [2], [3]. In addition, the topology of an ad hoc network can vary because of the characteristics of the radio channels. The limited radio propagation range of wireless devices imposes that the communication in MANETs is often multihop, which causes the node to work as a host or router. Moreover, bandwidth and battery power are limited. These constraints together with the dynamic network topology, make routing and multicasting in ad hoc networks extremely challenging [5]. As a result of the infrastructure-less nature of mobile networks, as well as their flexibility and low cost, such networks have had widespread applications in recent years, such as disaster recovery, rescue missions, military operations in a battle-field, conferences, and outdoor entertainment activities. One-to-many or many-to-many communications is one of the most important natures of these applications. To use unicast transmission in such applications imposes multiple signaling, which has high cost in MANETs. Instead, multicast approach is the highly preferred choice, in which, one signaling to many receptions can efficiently save node resources and network bandwidth. Therefore, multicast routing can be applied efficiently to construct group communications to deliver messages from a sender to multiple receivers. The difference between the natures of traditional wired and wireless networks prevents the application of the same multicast routing protocols. For this purpose, many multicast routing protocols have been designed to meet MANET characteristics.
Generally, multicast routing protocols can be classified based on how the routing structure is constructed and maintained; these classifications are tree-based and mesh-based routing protocols [4]. Tree-based routing protocols construct either a shared-tree that connects all multicast members or a sourcebased tree that is established and maintained for each source node of a multicast group. In tree-based protocols, only a single path will be available to serve data packets that are forwarded from source to receiver. However, the mesh-based approach provides robustness in the case of node mobility, but it requires increasing the flooding through a mesh. This condition results in inefficient bandwidth usage, high overhead, and lack of management of traffic load. The tree-based approach is considered efficient in terms of traffic distribution and has a low overhead. The disadvantage of the tree-based approach is low reliability under high speed, in which link failures trigger a reconfiguration of the entire tree as mobility increases [17]. To apply an efficient approach in terms of traffic distribution and robustness, we propose a scheduled-links mechanism for multicast routing (SLMR) in MANETs. SLMR is an improvement approach to source tree-based routing. To achieve robustness and reliability in the presence of node mobility, SLMR provides multiple node-disjoint paths between a source-receiver pair. Instead of using all these paths simultaneously, where they may interfere with each other, a scheduling scheme has been introduced to support efficient usage of network resources. As a result of applying scheduling for discovered paths, a load-balance metric has been derived from each path, which enables management of traffic load across scheduling paths.

The main contribution of this paper is to propose a new multicast routing protocol based on mobility prediction in MANETs. In this new approach, one control signaling will be used to con- 
struct and schedule multiple paths to the receiver. The routes to serve data packet forwarding are discovered and scheduled based on the cooperation process between sources and receivers. The set of discovering paths will be scheduled to support load balance and traffic distribution between these paths, as well as reduce congestion and save network resources. Load balance and traffic distribution is achieved in SLMRP through controlling path utilization time for each source-receiver pair. Path utilization time is controlled by computing multicast routing activation timers (MRATs) and path timeout timers (PTTs) according to the set of route expiration time for their paths. To increase reliability and robustness, which relies on the set of discovering paths, SLMRP route discovery mechanism ensures that these paths are loop-free and node-disjoint.

The rest of this paper is organized as follows:

Section 2 highlights some multicast routing protocols, where numerous approaches have been proposed to address and overcome obstacles during the implementation of multicast routing protocols in MANETs. Section 3 presents system models in the SLMR algorithm. Section 4 shows the operation of SLMRP, the route establishment, and maintenance. Section 5 analyzes the SLMR algorithm and proves that SLMRP discovered paths are loop-free and node-disjoint. Section 6 describes the performance comparison results between SLMRP, MAODV [6], PUMA [7], and PMRP [16]. Our comparison evaluates the experiments to test the performance of our proposed scheme against that of three different approaches in multicast routing, namely, mesh-based, tree-based, and mobility prediction. The comparison study uses different metrics to examine the effect of node mobility and traffic load on protocol performance. Finally, Section 7 presents the conclusion.

\section{Related work}

Up to date, many multicast routing protocols have been proposed and implemented for MANETs. The multicast ad hoc on demand distance vector protocol (MAODV) [6] is considered a classical example of a shared tree-based routing protocol. MAODV is an extension of ad hoc on demand distance vector (AODV) [8] unicast protocol. MAODV utilizes a similar discovery process of AODV to acquire routes toward the group on demand. The protocol provides and maintains a shared tree and single path for each multicast group, including relays and receivers. In MAODV, link break detection needs continuous periodic neighbor sensing and a periodic "group hello message" for multicast forwarding state creation. The adaptive demand-driven multicast routing protocol (ADMR) [9] is a source tree-based routing protocol. ADMR constructs and maintains a multicast tree for each sender where a single path serves forwarding data packet. ADMR eliminates the use of periodic network-wide floods of control packets, periodic neighbor sensing, and periodic routing table exchange. The joining operation of a new receiver to a multicast tree requires multicast solicitation as a network flood. Any source that receives this packet replies to the solicitation. In turn the receiver sends a join packet back to the source. A group member detects a disconnection from the multicast source in case of failure to receive a number of successive keep-alive packets.

On-demand multicast routing protocol (ODMRP) [10] is a mesh-based routing protocol. A periodic network-wide flood approach has been applied in mesh construction and maintenance in ODMRP. When a multicast source has data packets to send, that source starts a periodic Join-Request broadcasting to the entire network. This transmission constructs a mesh and repairs the link breaks that may occur between two JoinRequest intervals. Based on the receiving of a Join-Request at the receivers, Join-table includes route information that is periodically broadcasted toward the source. The selected nodes between the source and receivers will set a forwarding flag (FG-Flg) to build a mesh of nodes to forward data packets.

To reduce overhead, DCMP [11] attempts to select certain senders as cores to reduce the signaling by reducing the number of senders that achieve periodic broadcasting. A receiver-initiated and core-assisted approach has been introduced in CAMP [12]. In CAMP, one or more senders are utilized as a core for a multicast group. Receivers use unicast join requests toward a core to join a multicast group, which imposes a core pre-assignment and the existence of a unicast routing protocol.

The protocol for unified multicasting through announcements (PUMA) [7] is a mesh-based and receiver-initiated routing protocol. PUMA is introduced to improve the drawbacks of CAMP. PUMA applies a distributed algorithm to elect one of the receivers of a group as a core. This approach eliminates the need to pre-assign a core. For mesh construction and maintenance, a periodic multicast announcement signaling is used, which provides multiple routes from multicast sources to receivers. The limitation of PUMA is that all nodes must receive periodic signaling packets regarding each multicast group, regardless of the interest nodes may have in the group [13]. The performance comparison study in Section 7 shows the effect of this limitation on PUMA performance under different scenarios.

ODMRP represents the state-of-art for mesh-based multicast routing protocols. Many approaches have recently been presented to attempt to enhance the performance operation of this protocol. On-demand multicast routing protocol with efficient route discovery [14] has been introduced to improve the performance and efficiency of ODMRP. This protocol is a limited flooding version of ODMRP, which attempts to manage the flooding scheme by preventing contributing nodes from flooding Join-Query messages if the delay requirements are not satisfied. The contributing nodes have been modeled as M/M/1 queuing systems.

Another effort to reduce the excessive control overhead of ODMRP has been achieved by level-based on demand multicast routing protocol (LODMRP) [15]. In the proposed LODMRP scheme, the distance to the source node is cached to set up broadcasting level of Join-Q message. Therefore, each node controls the broadcasting of the Join-Q message based on the recorded distance to the source. The threshold for dropping the Join-Q message is the number of hops from the source to that node. 
Mobility prediction has been applied in the power-aware multicast routing protocol (PMRP) [16]. The protocol utilizes power and link expiration time as metrics to select the route to destination. PMRP employs the routing paths with the longest route duration of time to establish a reliable multicast tree [16]. PMRP discovers a single path between the multicast source and receiver. We will show that such scheme has a high probability of link breakage. Moreover, the high frequency of rediscovering operations caused by broken links consumes battery power and wastes scarce radio resources. The PMRP repair process starts before a link break and does not provide a solution to address a suddenly changing in nodes' speed or direction.

\section{System models in SLMR algorithm}

\subsection{Mobility prediction and packet formats}

Each node unitizes the location information of the corresponding GPS [18] to predict mobility parameters (i.e., location coordinates, speed, and moving direction). Two important assumptions are considered in this model. The first is a free-space propagation model [19], in which the signal strength depends solely on the distance to the transmitter. Also, Also, all nodes are synchronized with the GPS clock in the second assumption. Assume that nodes A and B are within the same transmission range $r$ of each other. The $\left(x_{1}, y_{1}\right)$ is the coordinate for mobile node $\mathrm{A}$, and $\left(x_{2}, y_{2}\right)$ is the coordinate for mobile node B. Let $v_{1}$ and $v_{2}$ be the mobility speeds, and $\theta_{1}$, where $\theta_{2}\left(0 \leq \theta_{1}, \theta_{2}<2 \pi\right)$, the moving direction. The amount of time that they will stay connected, $D_{t}$, is then predicted using [19].

$$
D_{t}=\frac{-(a b+c d)+\sqrt{\left(a^{2}+c^{2}\right) r^{2}-(a d-b c)^{2}}}{a^{2}+c^{2}}
$$

where

$a=v_{1} \cos \theta_{1}-v_{2} \cos \theta_{2}$

$b=x_{1}-x_{2}$

$c=v_{1} \sin \theta_{1}-v_{2} \sin \theta_{2}$

$d=y_{1}-y_{2}$

When $v_{1}=v_{2}$ and $\theta_{1}=\theta_{2}, D_{t}$ is set to $\infty$ without using the above equation. To obtain and utilize the information from GPS, the packets must include additional fields, as shown in Table 1. When a source node sends a request packet, it appends its location coordinates, speed, and moving direction in a request packet. The next hop neighbor of the source node who receives the request packet uses this information to predict the duration of time that they will stay connected according to their transmission range.

In the mobility prediction scheme, two parameters have been defined.

1. Link expiration time (LET): represents the duration of time that two nodes stay connected, which depends on their mobility parameters and transmission range, that is given in Equ. 1.

2. Route expiration time (RET): is equal to the minimum of

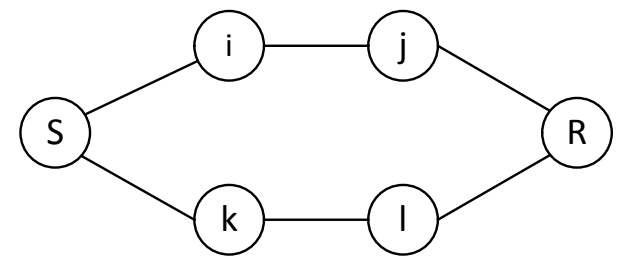

Figure 1: Example of MANET.

the set of LETs for the source-receiver routing path, as shown in Equ. 2.

$$
R E T=\min \left(L E T_{1}, L E T_{2}, \ldots, L E T_{n}\right)
$$

\subsection{Links scheduling with auto activation-deactivation mech- anism}

We adopt a novel mechanism for establishing and scheduling multipath multicast routes in MANET based on mobility prediction. The mechanism utilizes the available information of RETs at multicast receivers to compute two important timers; namely, MRAT and PTT as follows.

\section{Definition 3.1.}

For any $m$ paths available between multicast source-receiver pair, $P=\left\{p_{1}, \ldots, p_{m}\right\}$, with $R E T=\left\{R E T_{1}, \ldots, R E T_{m}\right\}$, such that $R E T$ is sorted in ascending order, MRAT for the $i^{t h}$ path in $P$ is given as follows.

$$
M R A T_{i}=R E T_{i-1} \quad i=2, \ldots, m
$$

where for path $p_{1}$ with minimum $R E T$

$$
M R A T_{1}=R E T_{0}=0
$$

MRAT represents the amount of time a scheduled path waits to change its state to be active and starts data forwarding. Equation (3) shows that MRAT depends on the RETs of the set of available paths for a source-receiver pair. From Equ. (3), $M R A T$ is equal to zero for the path that has a minimum value of RET (i.e., $p_{1}$ ), and becomes the first active forwarding path with no waiting time.

Suppose that the network is shown in Fig. 1, where two paths are available between multicast source $S$ and receiver $R$. For $p_{1}=\{S, i, j, R\}, R E T$ is equal to $R E T_{1}=\min \left(L E T_{S i}, L E T_{i j}, L E T_{j R}\right) . \quad$ In the same way, $p_{2}=\{S, k, l, R\}, \quad R E T_{2}=\min \left(L E T_{S k}, L E T_{k l}, L E T_{l R}\right) . \quad$ If $R E T_{2}>R E T_{1}$, then, $M R A T_{1}=0$, and $M R A T_{2}=R E T_{1}$. In the case of $R E T_{1}=R E T_{2}$, the receiver may select the path with the least number of activity or equally distributes traffic load among these two paths. At the moment when $p_{1}$ is deactivated and stopped forwarding data packets, the next path $p_{2}$ starts forwarding data packets for the multicast receiver. This process will continue for the available paths between the source and receiver. 
The path expires when the link with minimum LET value breaks. To force other links in the expired path to stop forwarding data packets, paths are deactivated by computing $P T T$, which is given in Equ. (4).

\section{Definition 3.2.}

For any $m$ paths available between multicast sourcereceiver pair, $P=\left\{p_{1}, \ldots, p_{m}\right\}$, with $R E T=\left\{R E T_{1}, \ldots, R E T_{m}\right\}$, where $R E T$ is sorted in ascending order, and $M R A T=$ $\left\{M R A T_{1}, \ldots, M R A T_{m}\right\}, P T T$ is defined as follows.

$$
P T T_{i}=\left(R E T_{i}-M R A T_{i}\right) \quad i=1, \ldots, m
$$

From (3)

$$
M R A T_{i}=R E T_{i-1}
$$

Then

$$
P T T_{i}=R E T_{i}-R E T_{i-1}
$$

Hence, for $p_{1}$

$$
\begin{gathered}
P T T_{1}=R E T_{1}-M R A T_{1} \\
M R A T_{1}=R E T_{0}=0
\end{gathered}
$$

Then

$$
P T T_{1}=R E T_{1}
$$

MRAT controls path activation mode, and PTT starts at the end of $M R A T$, which will be responsible for the deactivation of path forwarding status.

\subsection{Load balance technique}

We adopt traffic-based load balance technique, which is applied in links scheduling procedure. In this approach, one of two metrics can be used; namely, active path or traffic size. The active path metric defines the number of active routing path$\mathrm{s}$ that a node supports. Generally, a node that participates in forwarding packets to higher number of active routing path$\mathrm{s}$ will be more busier. The traffic size metric represents the total traffic load that exists at a node and its associated neighbors (measured in bytes) [20]. Both metrics can be applied in links scheduling process and according to the network administrator requirements. We introduce framework for applying an active path metric to select multicast routes between a source and a receiver that is subject to load balance. According to the scheduling mechanism that was described in (3.2), if $n$ paths have been scheduled to support forwarding data packets for a source-receiver pair, then each path $p \in n$ will be active for a duration of time $t_{i}^{a c t}$ given in Equ. (5).

$$
t_{p}^{a c t}=R E T_{p}-R E T_{p-1} \quad p=1, \ldots, n
$$

Any node in the network may support forwarding data packet$\mathrm{s}$ for more than one path in same time or in different periods of time depending on MRAT values. From (5), any node that supports $m$ paths can compute the current and future number of active paths for any given time $t$ within the activity duration $\left[t_{0}^{a c t}, t_{m}^{a c t}\right]$, which is equal to $\sum_{l=1}^{m} t_{l}^{a c t}$. A node can perform this task by accessing the cached information in a routing table as explained in section (4). However, we aim to determine the number of active paths for a limited duration of time, which is equal to the route lifetime for that path.

\section{Definition 3.3.}

For any node $j \in p$, we define $N_{j}^{a c t}$ as the number of active paths that node $j$ supports during the period of time that is equal to $R E T_{j}=\min \left\{L E T_{i_{1} s}, L E T_{i_{2} i_{1}}, \ldots . L E T_{j i_{n}}\right\}$, where $s$ is the source of path $p$, and set $i=\left\{i_{1}, i_{2}, \ldots, i_{n}\right\}$, is a set of upstream nodes to $j$, such that $i \in p$.

\section{Definition 3.4.}

For any path $p$ which contains $n-2$ nodes connected source $s$ to receiver $r, p=\left\{v_{1}: v_{1}=s, v_{2}, \ldots, v_{n-1}, v_{n}: v_{n}=r\right\}$, the active path cost function for $p$ is computed as shown in Equ. (6).

$$
C_{p}^{a c t}=\max \left\{N_{2}^{a c t}, \ldots, N_{n-1}^{a c t}\right\}
$$

(6) requires global information about $L E T$ at each node where different links have different $L E T$ values. We approximate computation of $C_{p}^{a c t}$ in a distribution fashion, as shown in the steps below.

Step 1. For any node $j \in p$, compute $N_{j}^{a c t}$ for the duration of time that is equal to $R E T_{j}$. We neglected to mention $s$ in $R E T$ notation where $R E T_{j}$ and $R E T_{j s}$ are identical.

Step 2. For any node $j+1 \in p$, if $R E T_{j+1}>R E T_{j}$, compute $N_{j+1}^{a c t}$ for the duration of time that is equal to $R E T_{j}$, then $C_{j+1}^{a c t}=\max \left\{N_{j}^{a c t}, N_{j+1}^{a c t}\right\}$.

Step 3. For Step2., if $R E T_{j+1}<R E T_{j}$, compute $N_{j+1}^{a c t}$ for the duration of time that is equal to $R E T_{j+1}$, then $C_{j+1}^{a c t}=\max \left\{N_{j}^{a c t} \times \frac{R E T j+1}{R E T_{j}}, N_{j+1}^{a c t}\right\}$.

The path activity cost metric provides a powerful feature design for the links scheduling process. This metric enables more choices to draw the current and future map of activation and deactivation of paths to avoid congestion and optimally distribute the traffic load among available paths.

\subsection{Reliability of scheduled links}

Route lifetime distribution of scheduled links can be expressed as follows. Let $P_{i}(t)$ be the probability for link $i$ to be broken at time $t$. Thus, the value of $\left(1-P_{i}(t)\right)$ represents the likelihood that link $i$ be available at time $t$. For route $R$ with $n$ links, the probability of $R$ to be broken at time $t$ is given using the following equation.

$$
P_{R}(t)=1-\prod_{i=1}^{n}\left(1-P_{i}(t)\right)
$$

Let $p=\left\{p_{1}, \ldots, p_{k}\right\}$ represents the set of scheduled paths that connected source $s$ to destination $d$. In the next sections, we prove that our proposed algorithm ensures that the paths in set $p$ are independent of each other. Therefore, the probability of set $p$ to be broken at time $t$ is given in Equ. 8 .

$$
P_{S c h}(t)=\prod_{j=1}^{k} P_{R_{j}}(t)=\prod_{j=1}^{k}\left\{1-\prod_{i=1}^{n}\left(1-P_{i}(t)\right)\right\}_{j}
$$


Despite the fact that the scheduled links in set $p$ are not activated at the same time, the reliability of $p$ is not affected, which is a powerful feature of the proposed algorithm that ensures reliability and efficient usage of network resources.

\section{Scheduled-links multicast routing protocol (SLMRP)}

\subsection{Overview}

In this section, we propose a SLMRP based on mobility prediction. SLMRP is an improvement of source tree-based routing, which is considered efficient in terms of traffic distribution. The source tree-based multicast routing is established and maintained for each source node of a multicast group. Multicast packet is forwarded along the most efficient path from the source node to each multicast group member. By contrast, mesh-based routing uses periodic signaling to establish multiple routes from the source node to receiver, in which a receiver may receive many copies of the same data packet via each route. This approach is considered more robust with the increasing link failures because of increasing nodes speed. The main limitation of mesh-based multicast routing is the dissemination of periodic signaling to maintain a mesh structure, which incurs considerable overhead. Thus, SLMRP utilizes location information to provide multiple routes to the receiver as in the mesh-based routing scheme. In the mesh-based routing scheme, the source uses all available paths at the same time to deliver packets to receivers, which results in inefficient bandwidth usage. Moreover, these routes are likely to interfere with each other. Therefore, we apply a scheduling process with auto activation-deactivation for these routes. This combination of two important features of source tree-based and mesh-based multicast routing makes SLMRP robust and efficient. Paths for a source-receiver pair will be auto activated and deactivated according to their RETs. The proposed algorithm addresses the link failure caused by suddenly changing in the speed or direction of a node in the network by providing redundant routes and a fast repairing mechanism. Load balance approach in SLMRP reduces the energy consumption of nodes in a network by managing the distribution of traffic load among available routes. In addition, the load balance scheme enhances network performance by avoiding congested routes, which results in high packet delivery ratio and reduces end to end delay.

Table 1: RREQ extra packet fields in SLMRP

\begin{tabular}{|l|l|l|l|l|l|l|}
\hline$x$-coord. & $y$-coord. & $v$ & $\phi$ & LET & DId & $N^{a c t}$ \\
\hline
\end{tabular}

\subsection{Route discovery phase}

Multicast session is established in SLMRP on demand, source initiated, and receiver-assisted for the paths scheduling process. As an on-demand protocol, SLMRP includes routing request and reply phases in route discovery operation. When a source has a data to send and does not have a route to receivers, a routing request packet (RREQ) is broadcasted throughout the entire network. A request packet, in addition to multicast session information, contains additional fields that belong to the mobility prediction scheme and load balance as illustrated in Table 1. The source uses GPS to compute current coordinates, movement speed, and direction to write in the RREQ packet. Furthermore, an intermediate node caches the previous hop ID (i.e., backward learning) when the routing request packet is received to use the ID in forwarding reply packets initiated by receivers. This node calculates the LET of the current link as in Equ. (1), then compares this value with the LET value of RREQ to choose the smallest value and rewrites in RREQ. In SLMRP, the source appends the value to zero in the LET field of the request packet. The source neighbors use hop count to detect the LET value, which has been written by the source. In addition, each node computes the number of active paths that node $\left(N^{a c t}\right)$ supports. Intermediate nodes perform this task by accessing a routing table that contains the information about scheduled paths and MART values that correspond to each path, as in Table 2. As mentioned Section (3.3), the intermediate node computes $C^{a c t}$ according to Equ. (6), appends this value in RREQ packet, and rebroadcasts RREQ.

In traversing along different paths, more than one request packet arrives to the receiver. When a receiver receives the first request packet, the receiver starts the paths scheduling process.

\subsection{Receiver-assisted paths scheduling process}

Upon receiving a first routing request packet, the multicas$\mathrm{t}$ receiver sets a waiting timer to receive many request packets. For each arriving packet during the waiting interval, the receiver computes RET and $C^{a c t}$. Also, the receiver records the SrcID, lasthopID, timestamp, and hop_count for this path. The receiver utilizes this recorded information in scheduling operation.

At the end of the waiting time, the receiver extracts a set of paths that satisfy the longest RET with least path activity requirements. The receiver computes two important timers in SLMRP; namely, the MRAT and PTT, which are given in Equ. (3) and (4) respectively. The receiver creates RREP packets and sends these packets back to the source along the selected paths. Each node receives RREP, processes it by setting MRAT and PTT timers to control activation and deactivation status. Also, this node caches the path information contained in RREP in the routing table, and then forwards RREP to the next hop in the same path. The routing table includes srcId, DstId, pathId, MART, PTT, and pathIdmax, as shown in Table 2. Fig. 3 shows the values of the MRATs and PTTs for the network of Fig. 2.

When a source receives RREP, this source establishes communication and sends data packets to the receivers, where only the current active paths forward data packets. The SLMRP discovery process is formulated in Algorithm 1.

\subsection{Waiting time}

The literature [21] investigated that the packets should be delivered to multicast receivers before the maximum threshold of $250 \mathrm{~ms}$. Moreover, the multicast receiver spends enough time to receive RREQs to ensure that the discovered paths set includes the most reliable paths in terms of link lifetime. Therefore, the 


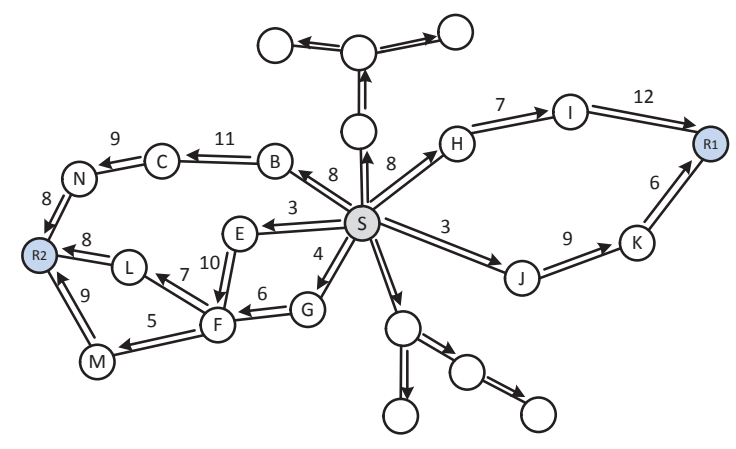

(a) Routing request phase

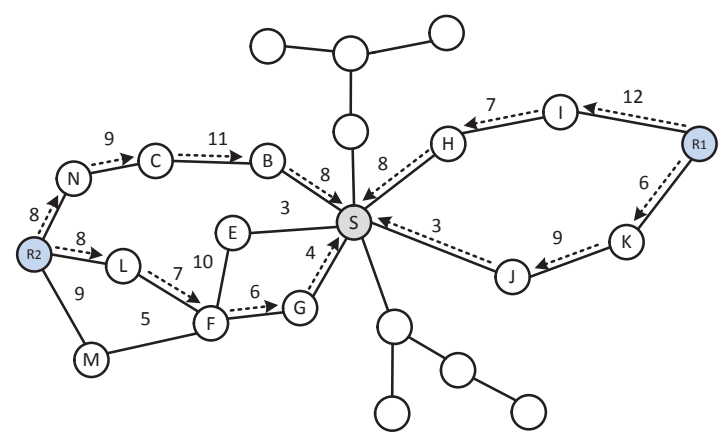

(b) Routing reply phase

Figure 2: Route discovery process.

scheduling process for these paths is applied after a certain time. To remove any encountered effect for this non-restricted model, we introduce a method to address the waiting time effect, by selecting a temporary path for forwarding data packets until scheduling process is established. In SLMRP, the effect of waiting time period, which is needed to accomplish the scheduling mission was eliminated. A temporary path to employ the role of delivery data packets is used until the receiver completes the sending replies along the selected paths. When the receiver receives the first RREQ, a RREPLY packet is initiated and sent back along that path. The MRAT for this path is set to be zero and PTT to the maximum waiting time, which is determined to be $250 \mathrm{~ms}$. We use a flag to inform the source that this is a temporary path with pathID is equal to zero. Upon receiving this reply, the multicast source will start sending data packets along this path until it receives new RREPLYs without flags. If the source found that the temporary path has been included in the scheduling paths by checking pathIDs' order, then it will do nothing, and the path will expire according to the formal PT$\mathrm{T}$ assigned in scheduling process. If it has not been included, then the source may use a piggyback packet to deactivate this path, where a formal path has entered the service and is active to deliver multicast data packets.

\subsection{Example}

The network shown in Fig. 2 is considered to explain the SLMRP operation. We focus on the paths scheduling process between source $S$ and receiver $R_{1}$. Multicast source node $S$ has data to send to receivers $R_{1}$ and $R_{2}$. The source $S$ creates RREQ, appends mobility parameters, and sets the LET's field in a request packet to zero. $\mathrm{S}$ broadcasts a RREQ packet to its neighboring nodes. Nodes $\mathrm{H}$ and $\mathrm{J}$ compute their LETs between themselves and source $S$ to append this information in the LET field after checking that hopcount is one. In addition to LETs, each node computes $N^{a c t}$ values, appends these values with mobility parameters to RREQ, and rebroadcasts it. In the same way, next hop nodes (i.e., I and K ) compute LET and compare it with a value of LET in the received request packet to choose the minimum value and append it with their mobility parameters and rebroadcast RREQ to its neighboring nodes.
As in (6) and steps (1-3) in Section (3.3), to simplify the explanation of the SLMRP scheduling process, we suppose for $p_{1}=\left\{S, J, K, R_{1}\right\}$ and $p_{2}=\left\{S, H, I, R_{1}\right\}$ that

- $N_{J}^{a c t}=1$ paths, computed for duration of $R E T_{J}=3$.

- $N_{K}^{a c t}=2$ paths, computed for duration of $R E T_{K}=3$.

- $N_{H}^{a c t}=2$ paths, computed for duration of $R E T_{H}=8$.

- $N_{I}^{a c t}=3$ paths, computed for duration of $R E T_{I}=7$.

and,

- $C_{S-H-I-R_{1}}^{a c t}=3$
- $C_{S-J-K-R_{1}}^{a c t}=2$

When $R_{1}$ receives the first RREQ, it waits to receive more than one RREQ packet, to compute MRATs and PPTs based on the recorded information during the waiting time. As we described in Section (4.4), $R_{1}$ may use $p_{1}$ or $p_{2}$ temporarily for a specific time to eliminate the delay caused by waiting time. Later, if this path is qualified to be in the forwarding set, then the receiver will include it during the scheduling process as a formal path. For $S-R_{1}$ pair shown in Fig. 2(a), the set $p=\left\{p_{1}, p_{2}\right\}$ has the longest $R E T=\{3,7\}$ with the least $C^{a c t}=\{2,3\}$. MRATs and PTTs for network in Fig. 2 are illustrated in Table 3, where we suppose for $S-R_{2}$ pair that $C^{a c t}=\{0,0\}$.

All PTTs values start at the activation mode, as shown in Fig. 3. Table 3 shows that the utilization time of each path (represented by PTT) is approximately equaled. SLMRP discovered paths that are independent of each other, which increases reliability and makes the protocol more robust. The receiver includes the proper MRATs and PTTs for each path in RREP packets and sends RREPs back to the multicast source node. Nodes in any selected path read MRAT and PTT in RREP to set timers to determine the activation and deactivation time for that path. The member node of the forwarding path may be active for a certain source and not active for another source based on scheduling duration. This approach will avoid congestion by reducing the uninteresting traffic in network. After source node $S$ receives RREP, it starts sending data packets. At first, 


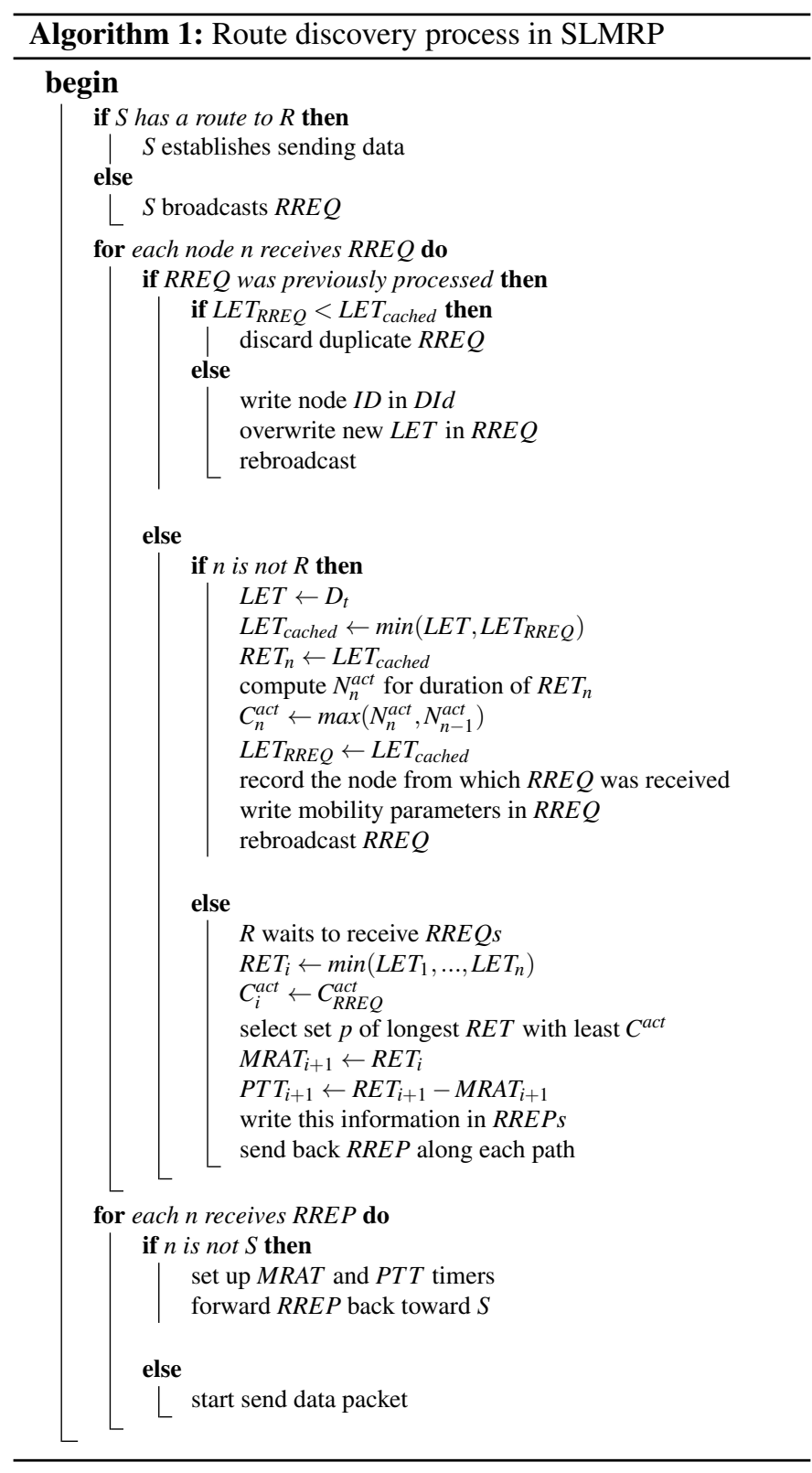

only the paths S-B-C-D-R $\mathrm{R}_{1}$ and S-J-L-M-R 2 are active and forward data packets. MRAT values for these two paths are equal to zero, as shown in Table 3. When these paths are deactivated, the next paths in the scheduled-paths set start forwarding data packets.

\subsection{Loop-free and node-disjoint paths set selection}

SLMRP discovers multiple scheduled paths, where the activation and deactivation of these paths are auto controlled by MRATs and PTTs. This method prevents a source node from having more than one active path to the intended receiver at the same time. Auto activation and deactivation based on path RET will be applied sufficiently in discovering loop-freed paths between multicast source and receiver node. If two paths with the same RET are discovered, then SLMRP selects the path with the least number of activity or equally distribute traffic load a-
Table 2: RREP extra packet fields in SLMRP.

\begin{tabular}{|l|l|l|l|l|}
\hline PathIDmax & PathID & RET & MRAT & PTT \\
\hline
\end{tabular}

Table 3: MRATs \& PTTs for S-R $1, \mathrm{~S}_{2} \mathrm{R}_{2}$ in Fig. 2

\begin{tabular}{|c|c|c|c|c|}
\hline Table 3: MRATs \& PTTs for S-R & S-R 2 in Fig. 2 \\
\hline \hline S-J-K-R 1 & RET & $C^{a c t}$ & MRAT & PTT \\
\hline S-H-I-R 1 & 3 & 2 & 0 & 3 \\
\hline S-G-F-L-R 2 & 4 & 0 & 0 & 4 \\
\hline S-B-C-N-R 2 & 8 & 0 & 4 & 4 \\
\hline
\end{tabular}

mong these two paths.

In the same way, node-disjoint paths are a natural result of applying the scheduled-paths algorithm based on RET. The idea behind selecting a set of node-disjoint paths is to increase the reliability of this set of discovering paths, where the failure of paths will be independent of each other. As we will explain in the route maintenance operation in SLMRP, the failure of any link on one path can be detected and corrected by the activation of the next path in the scheduled-paths set and resuming data transmission through that path. To select a set of nodedisjoint paths in the route discovery process, SLMRP uses simple and low-cost procedure to ensure that this set of paths is node-disjoint. The procedure comprises of a node smart duplicate forwarding and receiver-assisted paths scheduling process. Each node forwards RREQ and caches LET (it represents the RET of a path from the source to this node), previous hop ID, and source ID. When it receives a duplicate copy of RREQ, it checks if LET is greater than the cached value, overwrites the new information, and appends its ID in the disjoint field (DId) in extra fields shown in Table 1 and forwards RREQ. If a duplicate LET is less than the cached LET, the node drops this packet. At the receiver and during waiting time, and according to its receiver-assisted operation, this receiver records information to use it in scheduling calculation. If it receives a duplicate copy with the same previous hop and new node DId field in the RREQ packet, then it checks the greatest value of RET between duplicate copies received from last hop node and records all information relative to this path. This procedure will remove path overlap at a certain node regardless of whether it is applied for one last hop or many last hops to the receiver. In the more than one last hop scheme, the receiver will receive redundant copies tagged with an extra node DId field, which means that these last hops locate on paths overlap at a same node. Between all these overlapped paths, the receiver selects one path with the highest RET and sends RREP along this path. At the intermediate node, it forwards this RREP according to their cached information, which represents a single and reliable path.

For the network in Fig. 2(a), path S-E-F-L-R 2 overlaps with S-G-M-R $\mathrm{R}_{2}$ at node F. To increase the reliability of our selecting paths set via discovering independent paths, SLMRP uses a simple and low-cost idea to achieve this scheme. When node $F$ receives the first RREQ from node $E$, for example, it calculates LET for link F-E and compares it with LET in the request packet. If the calculated LET is greater, then overwrites the new 


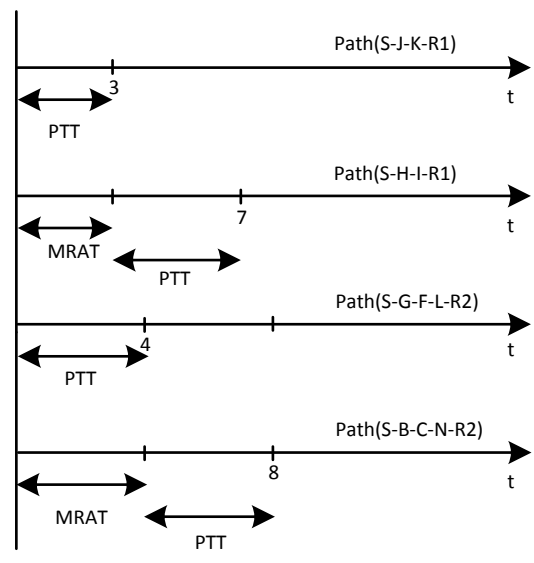

Figure 3: MRATs \& PTTs for network of Fig. 2.

information in RREQ and rebroadcasts it. Also, node F caches the previous hop Id and LET, which was written in RREQ, before it rebroadcasts it. If $\mathrm{F}$ receives a duplicate request packet copy from node $\mathrm{G}$, then it makes the same calculation as in the first request packet. Now, node F compares the LET for path F-G-S with the cached value for path F-E-S, and if the second one is greater, then it removes the cached information and replaces it with a newer value of path F-G-S and appends its ID in the request packet DId field. In the receiver-assisted process, when a receiver receives two copies of RREQ from same last hop nodes (i.e., $\mathrm{L}$ and $\mathrm{M}$ ) with an extra field in the second copy, which represents the node ID where paths overlap occurs. In such case, the receiver $R_{2}$ selects the reliable path through node $\mathrm{L}$ to send RREP. Node $\mathrm{F}$ caches the information of the reliable path. Thus, it forwards RREP via node G. Finally, path S-GF-M- $\mathrm{R}_{2}$ is added to the scheduled-paths set as shown in Fig. 2(b).

\subsection{Route maintenance process}

\subsubsection{Multicast join operation (source-assisted paths schedul-} ing process)

When a node decides to join a multicast group, it simply broadcasts a join request packet (JREQ) throughout the entire network. It uses extra packet fields to append its mobility parameters. According to the path scheduling mechanism in SLMRP, only a node that is a source of a multicast group may respond. If a node receives a join request packet to a multicast group that is not a member, then it calculates LET and $N^{a c t}$ as in RREQ process and creates a reverse route entry to the previous hop node and then broadcasts the join request packet to its neighbors. The member in a multicast group forwards this request to the source node. In the join operation, the multicast source assists in scheduling the routes to the intended node in the same way that is applied as in case of route discovery, where the receiver assists in the paths scheduling process. At the end of the waiting time and recording available paths information,

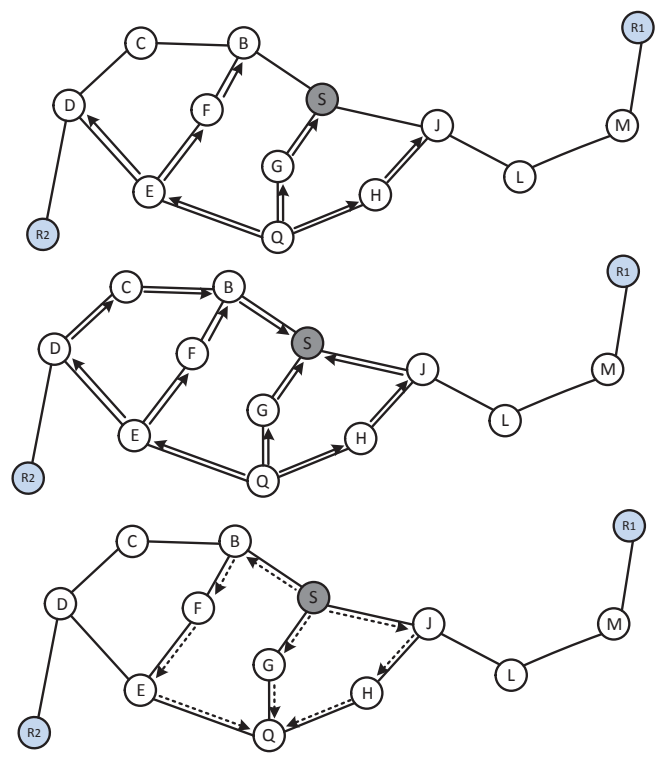

Figure 4: Node join operation.

the source sends back a join reply (JREP) packet to the perspective node, including MRAT and PTT of the selected path. Fig. 4, shows the multicast join operation. Node $\mathrm{Q}$ broadcasts JREQ to join the multicast group. Nodes E, H, F, and G are not members, where they append mobility parameters and rebroadcast packet to their neighbors after calculating LET and $N^{a c t}$, Multicast group member nodes D, C, B, and J forward JREQ to source $\mathrm{S}$, which in turn sends back JREP. In JREP traversing back toward node $\mathrm{Q}$, if a node is a group member and active, then it will not change its MRAT. By contrast, if the node is not active, it selects a minimum value of MRAT (current MRAT and included in JREP) and changes its state to active to start forwarding data packets from the multicast source. On other hand, this node should select maximum PTT value to control the active state for this path to Q. During the waiting time period, the source uses the same mechanism in the route discovery process to deliver data packets to $\mathrm{Q}$.

\subsubsection{Node pruning operation}

When a node selects to leave the multicast group, if a pruning node is a receiver, then it simply sends a Prune Message (PM) packet to its upstream node. When the upstream node receives the PM packet, if it becomes a leaf node, then it removes the corresponding entry from its multicast routing table and resets PTT and stops forwarding data packets. Also, it can further prune itself and in turn sends a PM packet to its upstream node. As a result of this process, this path will be removed from the multicast paths set. More than one path can be removed in the same way if multiple paths exist between this receiver and the multicast source.

\subsubsection{Turn between routes and local repair due to broken link}

Link break easily occurs in MANETs because of the mobility of nodes. SLMRP based on mobility parameters can detect the 


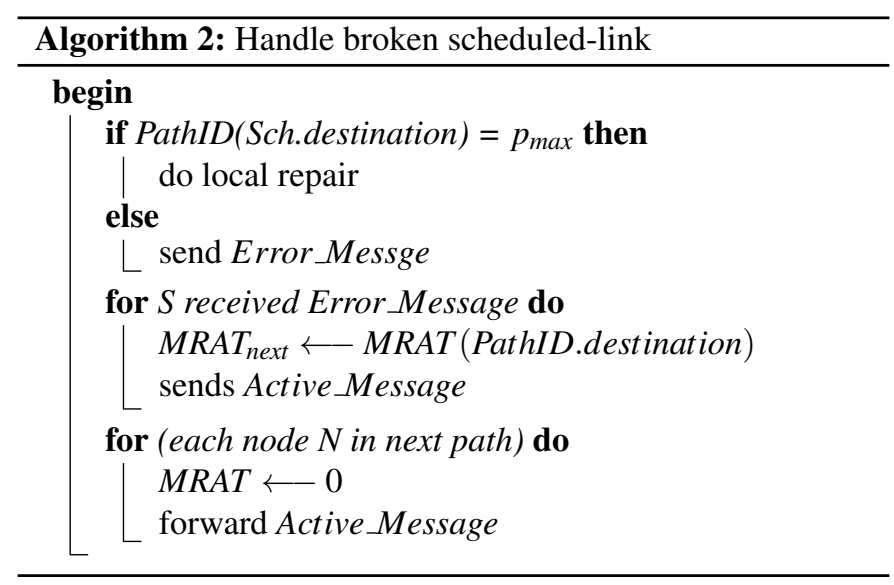

time when the link will break. In this case, the next path in the scheduled-paths set will start forwarding data packets according to the SLMRP activation scheme. Although the probability of an unexpected link break still exists. A sudden change in the node speed or direction of movement causes the computed LET value of that link to be different from the real LET. In such cases, the upstream node in the route detects link break by failure to receive a duplicate packet from the next hop in the active path. SLMRP deals with pruning of a multicast non-receiver member as a link break and applies turn between routes mechanism to handle such cases. The difference in pruning is that the upstream node detects link failure by receiving a pruning message PM. In such a failure, if the cached pathId at upstream node is smaller than PathIdmax, then it sends ErrorMessage back to the source. The source in turn sends Active_Message along the next path to reset MRAT and starts forwarding data packets to the receiver. If no other path is available to the receiver, where the upstream pathId is equal to PathIdmax, then this node will try to repair this link by sending a local request. If the local repair failed to repair this link, then the upstream node informs the source by sending back Error_Message, where the source initiates a new route discovery process. Algorithm 2 is used to decide if the upstream node uses a turn between routes or to perform local repair.

\section{Analysis}

We show that no loops can be found during the node forwarding process. At the same time, discovered routes in SLMRP are node-disjoint. Let $G(V, E)$ be an undirected weighted graph, where $V$ is the mobile nodes and $E$ is the wireless links between them. The link cost equals the link LET between the connected nodes pair.

\section{Definition 5.1.}

Paths $P_{d i s_{1}}=\left\{s, a_{1}, a_{2} \ldots, d\right\}$ and $P_{d i s_{2}}=\left\{s, b_{1}, b_{2}, \ldots ., d\right\}$, which connect the source $s$ to destination $d$ are node-disjoint paths if they do not have any common node, except the source and destination, such that $P_{d i s_{1}} \cap P_{d i s_{2}}=\{s, d\}$.

In the next lemmas and theorem, we use values described in Table (4).
Table 4: Valuse in proof

\begin{tabular}{ll}
\hline Value & Description \\
\hline$R R E Q_{n}^{d}$ & duplicate $R R E Q$ received at node $n$ \\
$L E T_{n}^{c}$ & cached $L E T$ at node $n$ \\
$L E T_{n}^{c_{r}}$ & computed $L E T$ value due to receiving $R R E Q_{n}$ at $n$ \\
$L E T_{n}^{c_{r d}}$ & computed $L E T$ value due to receiving $R R E Q_{n}^{d}$ at $n$ \\
$L E T_{i j}$ & $L E T$ value of the link connects $i$ to $j$ \\
$R T$ & retransmission table \\
\hline
\end{tabular}

Lemma 1. For any upstream node $i$, the condition $L E T_{i}^{c_{r d}}<=$ $L E T_{i}^{c}$ is sufficient to drop $R R E Q_{i}^{d}$ from downstream node $j$, if and only if $j$ has only one upstream adjacent vertex $i$.

Proof. For downstream node $j$, let $L E T_{j}^{c}=L E T_{j}^{c_{r}}=$ $\min \left(L E T_{i}^{c}, L E T_{i j}\right)$. When the upstream node $i$ receives $R R E Q_{i}^{d}$ from $j$ due to retransmission of $R R E Q$, it processes $R R E Q_{i}^{d}$ as follows. Since $L E T_{i}^{c_{r d}}=\min \left(L E T_{j i}, L E T_{j}^{c_{r}}\right)=$

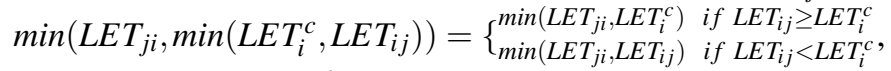
thus for all cases, $L E T_{i}^{c_{r d}}<=L E T_{i}^{c}$. This condition is sufficient for the upstream node $i$ to detect $R R E Q^{d}$ from downstream node $j$ and drop it successfully only based on $L E T$ values.

Lemma 2. For any downstream node $j \in P_{\text {dis, }} j$ will forward a duplicate copy RRE $Q^{d}$ from node $k:\{k$ is a node-disjoint $\}$ if $L E T_{j k}>L E T_{k i}$, where $i$ is any upstream node to $k$.

Proof. For the downstream node $j$ to the node-disjoint $k$, let $L E T_{j}^{c}=\min \left[\min \left(L E T_{i_{1}}^{c}, L E T_{k i_{1}}\right), L E T_{j k}\right]$, where $i_{1}$ is any upstream node to $k$. When node $k$ forwards a duplicate copy of $R R E Q^{d}$ received from other upstream node $i_{2}$, where $i_{1} \neq i_{2}$, then $L E T_{j}^{c_{r d}}=\min \left[\min \left(L E T_{i_{2}}^{c}, L E T_{k i_{2}}\right), L E T_{j k}\right]$. Node $j$ will check if that $L E T_{j}^{c_{r d}} \leq L E T_{j}^{c}$, which can result in cases:

Case1: $\quad L E T_{j k}>\min \left(L E T_{i_{2}}^{c}, L E T_{k i_{2}}\right)$, yields also $L E T_{j k}>\min \left(L E T_{i_{1}}^{c}, L E T_{k i_{1}}\right)$ since $\min \left(L E T_{i_{2}}^{c}, L E T_{k i_{2}}\right)>$ $\min \left(L E T_{i_{1}}^{c}, L E T_{k i_{1}}\right)$. Therefore, the condition $\min \left(L E T_{i_{2}}^{c}, L E T_{k i_{2}}\right) \leq \min \left(L E T_{i_{1}}^{c}, L E T_{k i_{1}}\right)$ will not satisfy and $j$ forwards $R R E Q^{d}$ received from $k$.

Case 2: $\min \left(L E T_{i_{1}}^{c}, L E T_{k i_{1}}\right)<L E T_{j k}<\min \left(L E T_{i_{2}}^{c}, L E T_{k i_{2}}\right)$, thus $L E T_{j k} \leq \min \left(L E T_{i_{1}}^{c}, L E T_{k i_{1}}\right)$ will not satisfy and $j$ forwards $R R E Q^{d}$ received from $k$.

Case 3: $L E T_{j k}<\min \left(L E T_{i_{1}}^{c}, L E T_{k i_{1}}\right)$, therefore, $L E T_{j}^{c_{r d}} \leq L E T_{j}^{c}$ satisfies, and node $j$ drops $R R E Q^{d}$ received from $k$.

Note that for Cases $1 \& 2$, when $k$ receives $R R E Q^{d}$ from $j$ due to retransmission of RREQ, LET $T_{k}^{c_{r d}}=\min \left(L E T_{j}^{c_{r d}}, L E T_{k}^{c}\right)=$ $\min \left[\min \left(\min \left(L E T_{i_{2}}^{c}, L E T_{k i_{2}}\right), L E T_{j k}\right), \min \left(L E T_{i_{2}}^{c}, L E T_{k i_{2}}\right)\right]$. Hence, $L E T_{j}^{c_{r d}}=\min \left(L E T_{i_{2}}^{c}, L E T_{k i_{2}}\right)$, and $L E T_{j k} \leq L E T_{j}^{c}$ satisfies. In such cases, node $k$ drops $R R E Q^{d}$ received from retransmission by $j$ to detect a reliable path from $i_{2}$.

Theorem 1 (Correctness of SLMR algorithm). If the SLM$R$ algorithm terminates when run on a connected network $G=(V, E)$ with a source $s$ and destination $d$, the multiple discovered paths connected $s$ to $d$ are scheduled 
and node-disjoint with a path cost is equal to $R E T_{P_{d i s}}=$ $\min \left\{L E T_{v_{1} v_{2}}, L E T_{v_{2} v_{3}}, \ldots, L E T_{v_{n-1} v_{n}}\right\}$, where $v_{1}=s$ and $v_{n}=d$.

Proof. Suppose that $i_{1}$ and $i_{2}$ are upstream nodes to node $k$. If $k$ received $R R E Q$ from node $i_{1}$, then $L E T_{k}^{c}=\min \left(L E T_{i_{1}}^{c}, L E T_{k i_{1}}\right)$. When $k$ receives $R R E Q^{d}$ from $i_{2}$, it calculates $L E T_{k}^{c_{r d}}=$ $\min \left(L E T_{i_{2}}^{c}, L E T_{k i_{2}}\right)$. The node $k$ checks if $L E T_{k}^{c_{r d}}>L E T_{k}^{c}$; in other words, $\min \left(L E T_{i_{2}}^{c}, L E T_{k i_{2}}\right)>\min \left(L E T_{i_{1}}^{c}, L E T_{k i_{1}}\right)$. Hence, from Lemmas 1 and 2, node $k$ forwards $R R E Q^{d}$ from $i_{2}$ if $L E T_{k}^{c_{r d}}>L E T_{k}^{c}$. When node $i_{1}$ receives $R R E Q^{d}$ from $k$ due to retransmission, it checks if $L E T_{i_{1}}^{c_{r d}} \leq L E T_{i_{1}}^{c}$, where $L E T_{i_{1}}^{c_{r d}}=$ $\min \left[\min \left(L E T_{i_{2}}^{c}, L E T_{k i_{2}}\right), L E T_{k i_{1}}\right]$. To avoid loop, it should be $\min \left[\min \left(L E T_{i_{2}}^{c}, L E T_{k i_{2}}\right), L E T_{k i_{1}}\right] \leq L E T_{i_{1}}^{c}$, while in this case, $\min \left(L E T_{i_{2}}^{c}, L E T_{k i_{2}}\right)>\min \left(L E T_{i_{1}}^{c}, L E T_{k i_{1}}\right)$.

In such cases, SLMR detects that case by using cached information in RT. In SLMR, $\forall v_{i} \exists R T: R T$ represents a set of neighbors from which $v_{i}$ has already received $R R E Q^{d}$. Then, in case of $L E T_{k i_{2}}^{r_{d}}>\min \left(L E T_{i_{1}}^{c}, L E T_{k i_{1}}\right)$ and $k \in R T$, node $i_{1}$ drops $R R E Q^{d}$ to prevent loop.

Lemma 2. Using SLMR, if a route $P_{\text {dis }}=\left\{v_{1}, \ldots, v_{i}, \ldots, v_{n}\right\}$ is found, where $v_{i}$ is the $i^{\text {th }}$ node on the path, $v_{1}=s$ is the source node (the sender of the RREQ) and $v_{n}=d$ is the destination, then $v_{i} \neq v_{j}$ for any $i \neq j$ and $1 \leq i, j \leq n$, i.e., no loop exist in this route.

Proof. As stated by Lemmas 1 and 2, and Theorem 1, any node $v_{i} \in P_{\text {dis }}$ cannot participate in other paths that span $s$ and $d$. Note that $v_{i} \neq s$ and $v_{i} \neq d$.

\section{Performance comparison}

\subsection{Performance evaluation}

We compare the performance of SLMRP against the performance of PUMA [7], MAODV [6], and PMRP [16], which are state-of-the-art multicast routing protocols for MANETs. PUMA and MAODV are both receiver-based protocols. However, PUMA is a mesh-based protocol and provides multiple routes from multicast sources to receivers. By contrast, MAOD$\mathrm{V}$ is a tree-based, multicast extension to AODV [8] unicast protocol. PMRP is a power-aware multicast routing protocol (PMRP) with mobility prediction. MAODV and PMRP provide only a single route between a multicast source and a receiver.

We compare SLMRP, PUMA, MAODV, and PMRP using Network Simulator 2 (NS-2 [22]). Table 5 shows the simulation environment parameters. The parameters of MAODV code are given in Table 6.

The performance metrics for comparison evaluation as follows:

1. Packet delivery ratio: The delivered data packets divided by the data packets expected to be delivered, which are the sent data packets times the number of multicast receivers.

2. End-to-end delay: The time when a data packet is sent by the source to the time the data packet is received at the receiver. 3. Control overhead: The transmitted control packets divided by the delivered data packets.
Table 5: Simulation environment

\begin{tabular}{|c|c|}
\multicolumn{2}{c|}{ Table 5: Simulation environment } \\
\hline Simulator & NS-2 \\
\hline Total nodes & 100 \\
\hline Simulation Time & 600 seconds \\
\hline Simulation area & $1000 \mathrm{~m} \times 1000 \mathrm{~m}$ \\
\hline Node Placement & Random \\
\hline Pause Time & 0 \\
\hline Mobility Model & Random Waypoint \\
\hline Radio Range & $250 \mathrm{~m}$ \\
\hline MAC Protocol & IEEE 802.11 \\
\hline Data source & CBR \\
\hline Data transmission rate & $2 \mathrm{Mbps}$ \\
\hline Data packet size & 512 bytes \\
\hline
\end{tabular}

Table 6: MAODV parameters

\begin{tabular}{|c|c|}
\hline allowed hello loss & 3 \\
\hline group hello interval & $5 \mathrm{sec}$ \\
\hline hello interval & $1 \mathrm{sec}$ \\
\hline my route timeout & $10 \mathrm{sec}$ \\
\hline bcast id save & $6 \mathrm{sec}$ \\
\hline active route timeout & $10 \mathrm{~s}$ \\
\hline rev route life & $6 \mathrm{sec}$ \\
\hline rreq retries & 3 \\
\hline route discovery timeout & $6 \mathrm{sec}$ \\
\hline rrep wait time & $500 \mathrm{~ms}$ \\
\hline
\end{tabular}

4. Total overhead: The total packets transmitted (control packets + data packets) divided by the delivered data packets. Total overhead shows the number of packets transmitted to deliver a certain number of data packets to the receivers.

\subsubsection{Simulation experiments}

To compare between SLMRP, PUMA, MAODV, and PMRP, we perform three experiments to determine the effect of mobility, traffic load, and group size on the performance metrics for each protocol. In experiment 1 , the node mobility speed varies across $\{0,5,10,15,20\} \mathrm{m} / \mathrm{s}$, the number of multicast senders is 5 , the receivers are 20 , the traffic load is set to $2 \mathrm{pkts} / \mathrm{sec}$, and multicast group is 1 .

In experiment 2 , the traffic load varies across $\{1,2,5,10\}$ pk$\mathrm{ts} / \mathrm{sec}$, the node mobility speed is $2 \mathrm{~m} / \mathrm{s}$, the multicast senders are 5 , the receivers are 20 , and as in experiment 1 , we set the multicast group to 1 .

In experiment 3 , the traffic load is $5 \mathrm{pkts} / \mathrm{sec}$, the node mobility speed is $10 \mathrm{~m} / \mathrm{sec}$, the multicast senders are also 5 , and the group size varies across $\{20,25,30,35,40\}$.

In each experiment, multicast senders and receivers are also mobile nodes and chosen randomly from among all the $100 \mathrm{n}$ odes in the simulation environment, and each result represents the average of 10 different random seeds.

\subsubsection{SLMRP vs. PUMA}

Figs. 5 and 6 show the performance of SLMRP and PUMA with respect to the packet delivery ratio, end-to-end delay, con- 


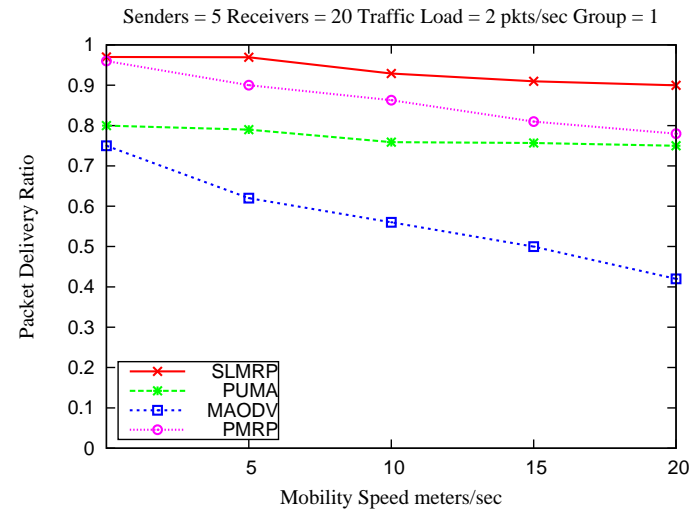

(a) Packet delivery ratio vs. mobility

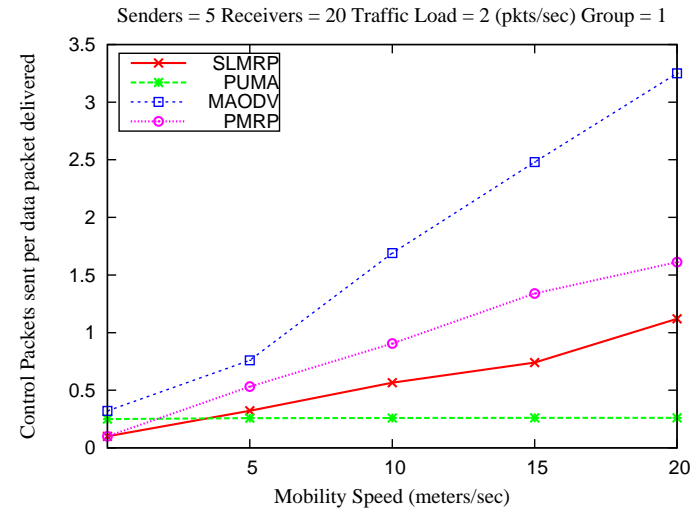

(c) Control overhead vs. mobility

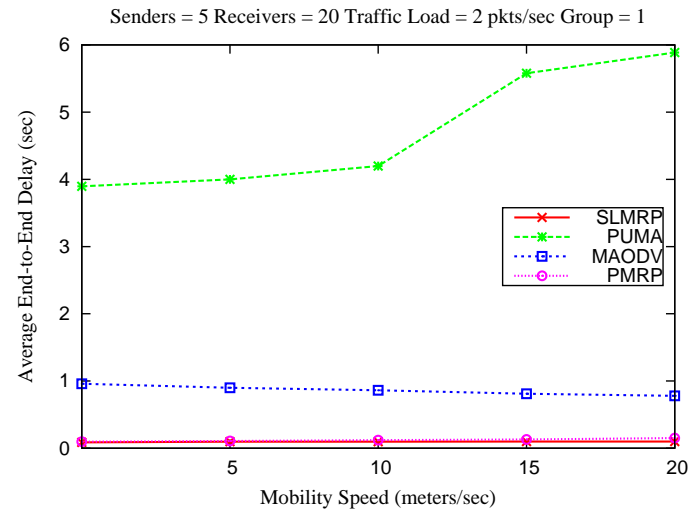

(b) End-to-end delay vs. mobility

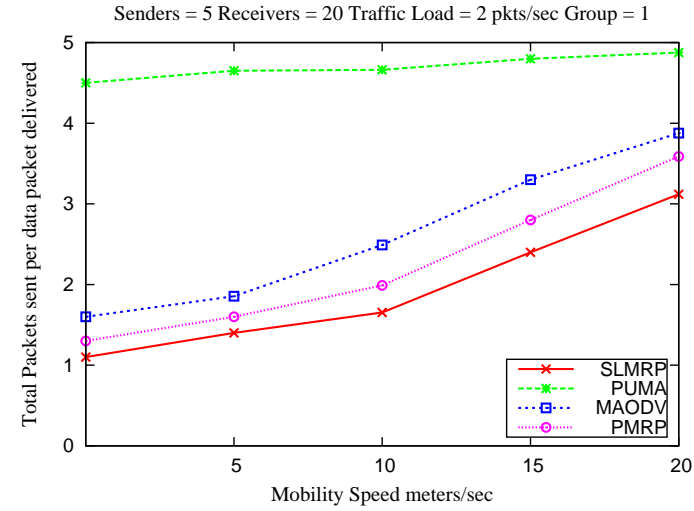

(d) Total overhead vs. mobility

Figure 5: Performance results for different nodes speed.

trol overhead and total overhead under different speeds and traffic loads scenarios. The SLMRP outperforms PUMA in the ratio of packets delivered to multicast receivers. PUMA has a high delivery ratio under low traffic load, as shown in Fig. 6(a), and as expected, PUMA appears to have constant control overhead in different scenarios based on the fact that it uses a constant interval to send a multicast announcement control packet. SLMRP has a lower overhead in different traffic load values as in Figs. 5(c) and 6(c). SLMRP uses node-disjoint paths, which reduces the effect of congestion under high traffic load. Also, using the node-disjoint paths reduces the transmission of control packets to overcome what appears to be link breaks due to loss of packets caused by collision. In high speeds, SLMRP incurs more control overhead to respond for links break. A careful analysis of the experiments shows that the control packet loss in PUMA under high traffic load due to collision highly reduces in packet delivery ratio, where PUMA doesn't respond for such cases. A dissemination of periodic signaling to nodes that have no interest in this traffic is considered as the main drawback of PUMA protocol, which significantly affects PUMA performance. We call this the "single-mode operation" of PUMA. This is accompanied by a corresponding decrease in packet delivery ratio and increase in packet delivery cost, which clearly appears in total overhead as shown in Figs. 5(d) and 6(d). This effect increases with an increase in traffic load, while it does not exhibit a significant change with the increase in node speed, where PUMA preserves good packet delivery ratio as in Figs. 5(a). Fig. 5(b) and 6(b) show the performance of the end-to-end delay under differen mobility speeds and traffic loads. SLMRP has lower end-to-end delay as a result of using a multiple scheduled path set with fast mechanism to activate the next path, thereby reducing the time for the construction and maintenance of these paths. Fig. 7(a) illustrates the packet delivery ratio under different multicast group sizes. The packet delivery ratio slightly decreases as multicast group size increases for both SLMRP and PUMA. However, the group size does not have a significant effect on the packet delivery ratio. PUMA approximately preserves constant control overhead under different multicast group sizes as shown in Fig. 7(b).

\subsubsection{SLMRP vs. MAODV}

Based on the simulation results shown in Figs. 5 and 6, the packet delivery ratio of MAODV is low in both two experiments compared with our proposed protocol SLMRP. At a low mobility speed, the multicast tree structure in MAODV is mostly static. When the node speed increases, the link break increases, and the single path in multicast tree becomes unstable. As traffic load increases, a significant congestion effect appears in the performance of MAODV, where a large number of packets are lost because of collision. In such cases, links appear to be 


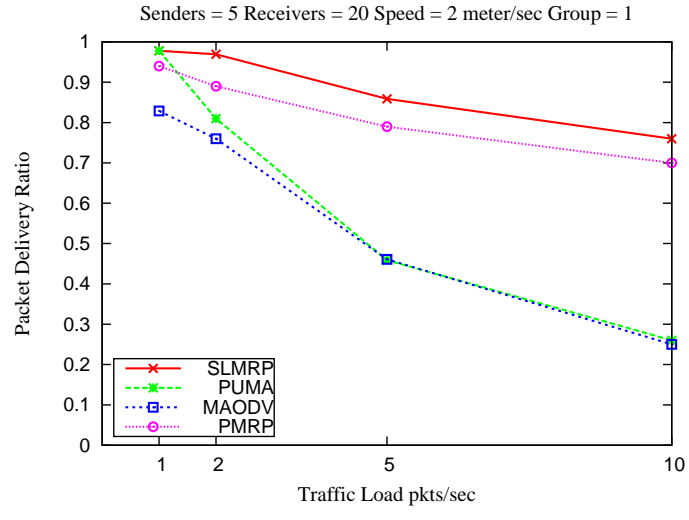

(a) Packet delivery ratio vs. traffic load

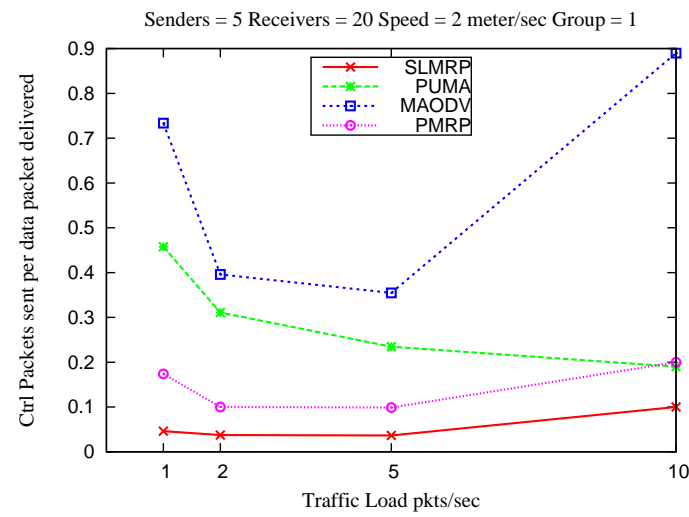

(c) Control overhead vs. traffic load

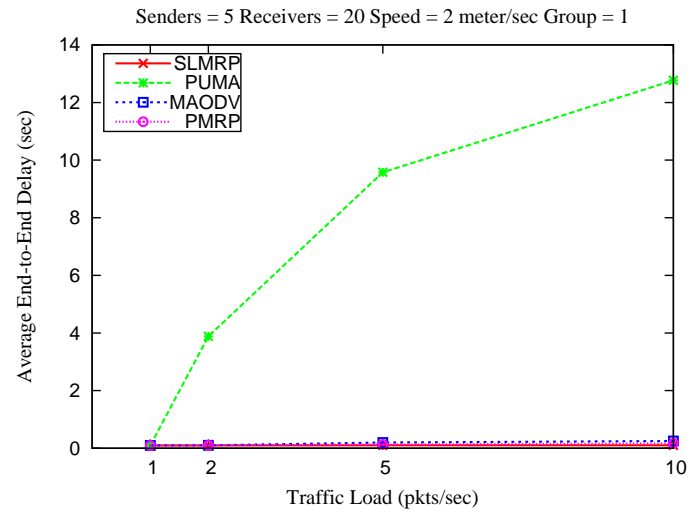

(b) End-to-end delay vs. traffic load

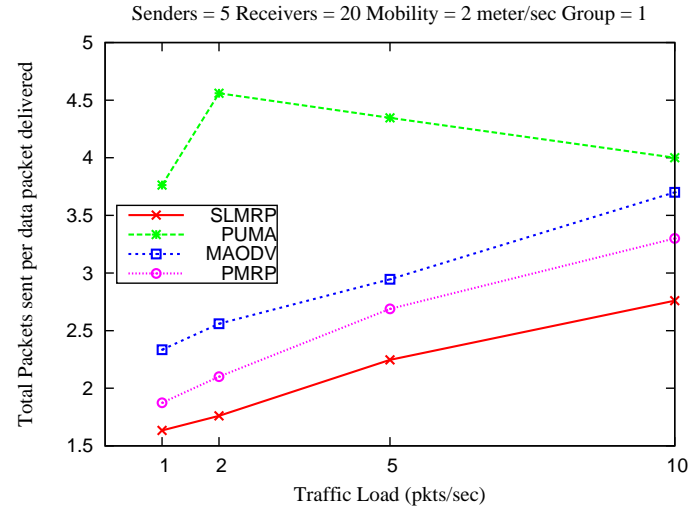

(d) Total overhead vs. traffic load

Figure 6: Performance results for different traffic loads.

broken, while in fact the drop was caused by collision. This condition causes the node to respond in MAODV and incurs significant overhead. As a result of using multiple scheduledpaths scheme, which is based on the computation of the link expiration time, the node can detect the time when the link will expire. The auto activation mechanism to the next path prevents any additional need to control packets in SLMRP, which has a significant effect on reducing congestion and packet dropping due to collision. Hence, we see that the SLMRP has a high packet delivery ratio under high traffic load and speed as in Figs. 5(a) and 6(a). Although MAODV uses a short path from the source to destination, thereby reducing the end-to-end delay, we can see from Figs. 5(b) and 6(b) that SLMRP has the same end-to-end delay for different speeds and traffic loads. This condition is due to the fact that SLMRP also chooses the minimum hops and lower time delay set among available paths. The existence of multiple paths with auto activation to the next paths in scheduled-paths set reduces the delay time needed to repair broken links. Link break in SLMRP occurs in case of a sudden change of the node speed or direction, where the likelihood of such a case is low in high mobility, although the LET is smaller than that at in lower speed. Using scheduled paths in SLMRP with a low likelihood of path break and turn to the next path scheme reduces control overhead as shown in Figs. 5(c) and 6(c). By contrast, MAODV uses three kinds of controlling packets to fix links that appear to be broken, i.e., RREQ, RREP, and MACT packets. Many RREQ packets may be broadcasted if a RREP packet is not received within a short enough time, thereby causing an increasing number of losses in hello packets due to collision. As a result of this condition, we can see the fast decrease in packet delivery ratio and fast increase in control overhead. The total overhead is shown in Figs. 5(d) and $6(d)$, where the cost of packet delivery of SLMRP is low with increasing mobility and has a small increase in cost in high traffic load compared with MAOD, where SLMRP may respond to preserve a high packet delivery ratio. MAODV performance degrades under increasing multicast group sizes as shown in Fig. 7 for the packet delivery ratio and control overhead.

\subsubsection{SLMRP vs. PMRP}

SLMRP and PMRP have similar characteristics as mobility prediction-based routing protocols. PMRP is a power-aware protocol that uses power metric to avoid routing over less energy nodes. PMRP does not provide a solution for managing traffic over routes for nodes that has power greater than the threshold value. This drawback affects the performance of the entire network, which works under PMRP. In Figs. 5(a) and 6(a) show that SLMRP has a higher delivery ratio than PMRP. SLMRP discovers multiple paths and uses these paths in the scheduling scheme to avoid the inefficient usage of network resources. 


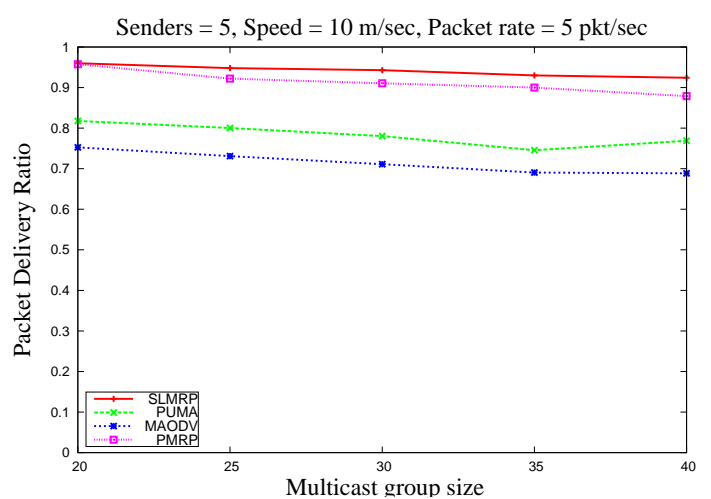

(a) Packet delivery ratio vs. multicast group size

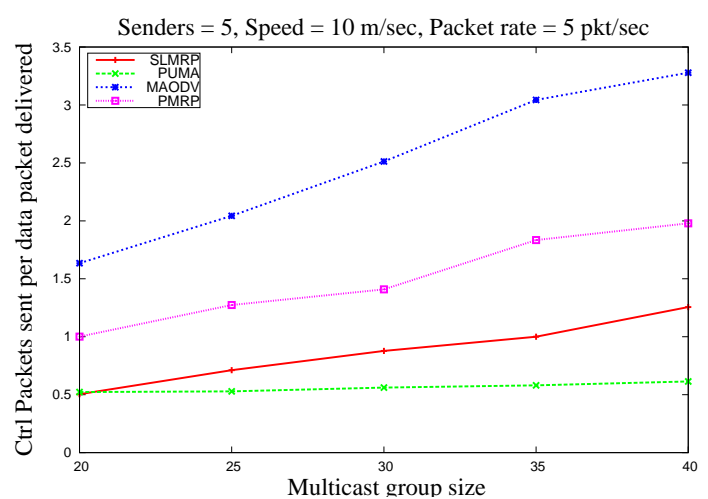

(b) Control overhead vs. multicast group size

Figure 7: Performance results for different multicast group sizes.

Reliable forwarding paths in SLMRP for each source-receiver pair reduces link breaks due to node mobility, which results in increasingly successful packet delivery. SLMRP eliminates the need for waiting time and produces more robust routes to reduce end-to-end delay, as shown in Figs. 5(b) and 6(b). Waiting time in PMRP has additional cost in terms of delay experienced during route discovery phase. Aside from that, PMRP depends on single path and with no mechanism to address sudden changes in speed or direction, while SLMRP can detect that condition without the need to rediscover or flooding-based repairing processes. This drawback is clearly appears in control overhead metric shown in Figs. 5(c) and 6(c), where PMRP incurs more control overhead under different scenarios. Furthermore, multicast source in SLMRP delivers the packet to the receiver with a total overhead cost that is less than that in PMRP as shown in Figs. 5(d) and 6(d). In Figs 7(a) and 7(b), SLMRP and PMRP encounters a decrease in the packet delivery ratio and increase in the control overhead as a response to an increase in the multicast group size, where SLMRP outperforms PMRP in both cases.

\section{Conclusion}

In this paper, we proposed SLMRP based on mobility prediction for MANETs. We used location information to select and schedule multipath set with auto activation and deactivation relative to paths' RETs to support a reliable and low-cost forwarding multicast data packets to receivers. The SLMRP discovery process ensures that the set of discovered paths is loop-free and nodes-disjoint paths, which increase reliability and protocol performance under different operation scenarios. Load balance strategy has been applied in SLMRP to reduce the effect of power consumption, increase of packet delivery ratio, and reduce overhead. Performance analysis and comparison of SLMRP against PUMA, MAODV, and PMRP show that SLMRP provides the highest packet delivery ratio, shortest end-toend delay, and lowest control overhead under different speeds and traffic load scenarios. In preserving a high delivery ratio under high speed, SLMRP incurred greater control overhead compared with PUMA. However, it reduces control overhead faces MAODV and PMRP single-path tree-based protocols. In future work can be extend SLMRP scheduling mechanism features to anticipate routes that will become available in the future and use it for route repair and load balancing.

\section{Acknowledgements}

This work was supported by the National Key Technology Research and Development Program of the Ministry of Science and Technology of China under grant no. 2012BAH93F01, the Innovation Research Fund of Huazhong University of Science and Technology under grant no. 2014TS095 and the National Science Foundation of China under grant no. 60803005.

\section{References}

[1] Subir Kumar Sarkar, T G Basavaraju, and C Puttamadappa. Ad Hoc Mobile Wireless Networks, Principles, Protocols and Applications. Taylor \& Francis, Boca Raton, USA, 2008.

[2] M. Younis and S. Z. Ozer. Wireless ad-hoc networks: Technologies and challenges. Wireless Commun. Mobile Computing, vol. 6, no. 7, pp. 889892, Nov. 2006.

[3] S. Guo and O. Yang. Energy-aware multicasting in wireless ad-hoc networks: A survey and discussion. Computer Commun., vol. 30, no. 9, pp. 2129-2148, June 2007.

[4] Luo Junhai, Ye Danxia, Xue Liu, and Fan Mingyu. A Survey of Multicast Routing Protocols for Mobile Ad-Hoc Networks. IEEE Communications Surveys \& Tutorials, vol. 11, no. 1, 2009.

[5] S. Bae, S.-J. Lee, W. Su, and M. Gerla. The Design, Implementation, and Performance Evaluation of the On-Demand Multicast Routing Protocol in Multihop Wireless Networks. IEEE Network, special issue on multicasting empowering the next-generation internet, vol. 14, no. 1, pp. 70-77, 2000.

[6] E. M. Royer and C. E. Perkins. Multicast Operation of the Ad-hoc On demand Distance Vector Routing Protocol. In: Proceedings of the IEEE ACM/IEEE MOBICOM, 1999, pp. 207-218.

[7] R. Vaishampayan, J. J. Garcia-Luna-Aceves. Efficient and robust multicast routing in mobile ad hoc networks. In: Proceedings of the IEEE MASS, Oct. 2004, pp. 304-313.

[8] C. E. Perkins, E. M. Royer. Ad-hoc on-demand distance vector routing. In: Proceedings of Second IEEE Workshop Mobile Computing Systems and Applications, pp. 90-100, 1999.

[9] J. G. Jetcheva, D. B. Johnson. Adaptive demand-driven multicast routing in multi-hop wireless ad hoc networks. In: Proceedings of the ACM MobiHoc, 2001, pp. 33-44. 
[10] S.-J. Lee, M. Gerla, and C.-C. Chiang. On-demand multicast routing protocol. In: Proceedings of the IEEE WCNC, 1999, vol. 3, pp. 1298-1302.

[11] S. K. Das, B. S. B. S. Manoj, and C. S. R. Murthy. A dynamic core based multicast routing protocol for ad hoc wireless networks. In: Proceedings of the ACM MobiHoc, 2002, pp. 24-35.

[12] J. J. Garcia-Luna-Aceves and E. L. Madruga. The core-assisted mesh protocol. IEEE J. Sel. Areas Commun., vol. 17, no. 8, pp. 1380-1394, Aug. 1999.

[13] J. J. Garcia-Luna-Aceves and Rolando Mechaca-Mendez. PRIME: An Interest-Driven Approach to Integrated Unicast and Multicast Routing in MANET. IEEE/ACM Transaction on Networking, vol. 19, no. 6, pp. 1573-1586, December 2011.

[14] Mohammad-Amin Kharraz, Hamid Sarbazi-Azad, and Albert Y. Zomaya On-demand multicast routing protocol with efficient route discovery. Journal of Network and Computer Applications, vol. 35, no. 3, pp. 942 950, May 2012.

[15] M. Ghasemi and M. Bag-Mohammadi. Level-based On-Demand Multicast Routing Protocol for Mobile Ad hoc Networks. In: Proceedings of the Wireless and Mobile Networking Conference (WMNC), 2013 6th Joint IFIP, pp. 1-7.

[16] Nen-Chung Wang, Jong-Shin Chen, Yung-Fa Huang, and Yu-Li Su. A Power-Aware Multicast Routing Protocol for Mobile Ad Hoc Networks With Mobility Prediction. Wireless Personal Communications, vol. 43, no. 4, pp. 1479-1497, December 2007.

[17] Rajashekhar C. Biradar and Sunilkumar S. Manvi. Review of multicast routing mechanisms in mobile ad hoc networks. Journal of Network and Computer Applications, vol. 35, no. 1, pp. 221-239, January 2012.

[18] E. D. Kaplan. Understanding GPS: Principles and Applications. Artech Hourse, Boston, MA, 1996.

[19] T. S. Rappaport. Wireless Communications: Principles and Practice. Prentice-Hall, Upper Saddle River, NJ, 1995.

[20] Chai Keong Toh, Anh-Ngoc Le, and You-Ze Cho. Load Balanced Routing Protocols for Ad Hoc Mobile Wireless Networks. IEEE Communications Magazine, vol. 47, no. 8, pp. 78-84, August 2009.

[21] Asif HM, Sheltami TR, and Shekshouki EE. Power consumption optimization and delay minimization in MANET. In: Proceedings of the 6th international conference on advances in mobile computing and multimedia (MoMM '08), November 2008.

[22] Fall K, Varadhan K (eds): The ns Manual. Available: www.isi.edu/nsnam/ns/ns-documentation.html 